\title{
The Influence of the Magnetron Sputtering Deposition Parameters on Optical Properties of a-C:H Thin Films
}

\author{
G. LAZ $\mathrm{AR}^{*}$
}

Bacău University, Calea Mărăşeşti 157, Romania

(Received January 17, 2001; revised version May 9, 2001)

\begin{abstract}
Tetrahedral, highly hydrogenated amorphous carbon films were deposited using rf sputtering of graphite by a magnetron sputter source in $\mathrm{Ar} / \mathrm{CH}_{4}$ atmosphere. The optical band gap, Urbach energy and refractive index of the deposited films were studied as a function of the bias voltage applied to the substrate during deposition and gas pressure. The films were characterized by visible spectroscopy. The variation of the optical band gap is considered as the result of the transition from unsaturated graphitic $s p^{2}$ bonds to tetrahedral diamond-like $s p^{3}$ bonds in the film. The variation of the refractive index is correlated with the density and optical band gap of the films.
\end{abstract}

PACS numbers: 78.66.Jg

\section{Introduction}

For a period of two decades there has been a continuous growth on the study of the deposition method and properties of amorphous carbon films. Initially, the interest in these thin films was as in a mechanical material that showed "diamond-like" properties. Currently, there is renewed interest in amorphous carbon, hydrogenated or nitrogenated, as an electronic material [1]. The ability to deposit these films at low temperature over a large area has also made them potentially attractive for applications in the area of optoelectronics.

The tetrahedral, highly hydrogenated amorphous carbon (a-C:H) films contain carbon atoms of both $s p^{3}$ and $s p^{2}$ configurations and their ratio varies on the deposition conditions. Although a detailed three-dimensional structure has not

*e-mail address: glazar@ub.ro 
been well elucidated, the energetically favorable $s p^{2}$ sites are believed to be segregated into sizable clusters and these are embedded in a $s p^{3}$ bonded amorphous matrix [2]. It was shown that the mechanical and optical properties of amorphous hydrogenated carbon depend strongly on the $s p^{3} / s p^{2}$ carbon ratio.

a-C:H has been prepared by plasma deposition from hydrocarbon gases [3-5], sputtering $[6,7]$, cathodic arc [8, 9], ion beam methods [10] and laser vaporization [11]. Common to all these methods is the deposition of a-C from particles whose energy is within the range of about $100 \mathrm{eV}$ up to several kilo-electron volts [5]. Carbon films prepared by much lower energy methods such as evaporation have been reported to be electrically conducting [12]. It appears that the most critical process parameter determining the film properties is the ion bombardment occurring during deposition [13]. In addition to several other techniques, which are capable of providing the requisite high energy density during film, deposition from hydrocarbon gases in rf discharges has become the most widely used [14]. During growth, the layers are subjected to ion bombardment controlled by the potential drop between the plasma potential and the negative dc bias voltage $\left(V_{\mathrm{b}}\right)$ at the film surface. With increasing bombarding energy the film transforms from a soft polymer-like $\left(V_{\mathrm{b}}<100 \mathrm{~V}\right)$ to a hard diamond-like $\left(100 \mathrm{~V}<V_{\mathrm{b}}<600 \mathrm{~V}\right)$ and finally to a soft graphite-like $\left(V_{\mathrm{b}}>600 \mathrm{~V}\right)$ material $[13,15]$.

The ion bombardment at the surface and processes in the plasma bulk can be separately controlled in a circular magnetron sputtering system. The magnetic field confines the electrons and improves gas decomposition and the bias voltage applied to substrate provides a selective control of the ion energies. The advantage of the magnetron sputter source is that this technique is widely established in industry and allows the deposition onto large areas with relatively high deposition rates. The use of suitable unbalanced magnetic-field configuration places the substrate immersed in the plasma so that the growing films is bombarded by energetic $\mathrm{Ar}$ ions from the plasma [7].

In this paper, the optical properties of highly tetrahedral a-C:H prepared by rf circular magnetron sputtering are presented. The optical gap, index of refraction and Urbach energy are investigated for different deposition conditions.

\section{Experiment}

a-C:H films were deposited by magnetron sputtering technique, using a pure graphite target and $99.99 \%$ pure $\mathrm{Ar} / \mathrm{CH}_{4}$ gas mixture. The magnetron installation, built in our laboratory in order to deposit thin films by dc and rf planar and circular magnetron sputtering, has been described previously [16-18]. In brief, the system consists in a cylindrical stainless-steel chamber with one magnetron (cathode) and two anodes. In an unbalanced magnetron, the plasma extends over both the target and the substrate, so that the Ar ions provide both the sputtering flux to the graphite target and the ion plating flux on the growing film. The plasma must 
also decompose the methane gas. A high flux of neutral $\mathrm{C}$ atoms is achieved by placing the magnets as close as possible to the graphite target. The configuration of the magnetic field is a critical parameter controlling the deposition rate and the ion plating intensity [7] and is a particular property of the magnetron.

In this work, the films were deposited by rf circular magnetron sputtering, using an rf source at $13.56 \mathrm{MHz}$. A stabilized dc power supply with maximum ratings $500 \mathrm{~V}$ and $240 \mathrm{~mA}$ was used for negative or positive substrate polarization. Films were deposited on glass substrates at a target-to-substrate distance of $3 \mathrm{~cm}$. The volumetric proportion between $\mathrm{Ar}$ and methane was maintained constant at 1:1. The pressure during deposition was between 0.2 and 0.01 Torr. During deposition the substrates are partially masked so that thickness measurements can be made by Tolansky instruments.

Optical absorption and reflection of the films was measured in the wavelength range 700-400 $\mathrm{nm}$ using an UV-VIS SPECORD spectrophotometer. The absorption coefficient $\alpha$ was calculated using Lambert's law and was explored as a function of photon energy. The optical band gap is determinate from a Tauc plot

$$
\sqrt{\alpha E}=G\left(E-E_{\mathrm{g}}\right) \text {. }
$$

The index of refraction is calculated from transmission interference spectrum in visible domain using a numerical method [19]. The values for hydrogen content and relative $s p^{3} / s p^{2}$ proportion were calculated from IR absorption spectra [20].

\section{Results and discussion}

Generally, with an applied bias voltage between $-200 \mathrm{~V}$ and $+400 \mathrm{~V}$ transparent and smooth films were obtained, colorless for pressures around 0.2 Torr and light yellow when the deposition pressure is lowered to 0.01 Torr. For a bias voltage below $-200 \mathrm{~V}$ the bombardment of the argon ions on the substrate is very strong and the color of deposited films is modified to a non-transparent dark brown. The high mechanical internal stress in carbon films reduces their adhesion on the substrate for films thickness over $1 \mu \mathrm{m}$, but — below these value - the adhesion on glass and silicon is very good. The proportion of tetrahedral-bonded carbon has a low variation for different deposition conditions and indicates a high tetrahedral structure. The hydrogen content between 47 and $55 \%$ shows a high hydrogenation of the films.

In Fig. 1 the transmission and reflection spectra in the range 28500$12500 \mathrm{~cm}^{-1}$ for a sample with thickness of $1.81 \mu \mathrm{m}$ are presented, obtained in the following conditions: working gas $\mathrm{Ar}: \mathrm{CH}_{4}=1: 1$, gas pressure $10^{-2}$ Torr and the substrate negative bias voltage $200 \mathrm{~V}$. Both spectra show clear interference patterns and indicate a good optical quality of the material.

Figure 2 shows the optical band gap of the films at various deposition pressures for two applied bias voltages. From this figure one can see that the optical 


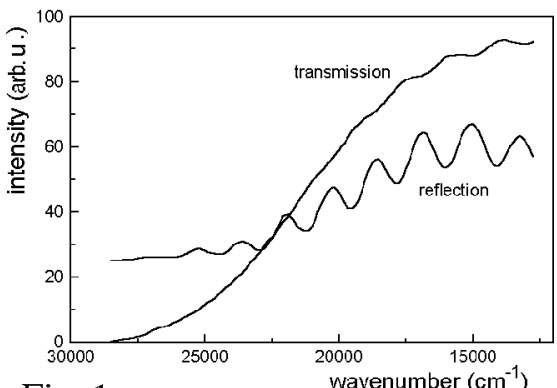

Fig. 1

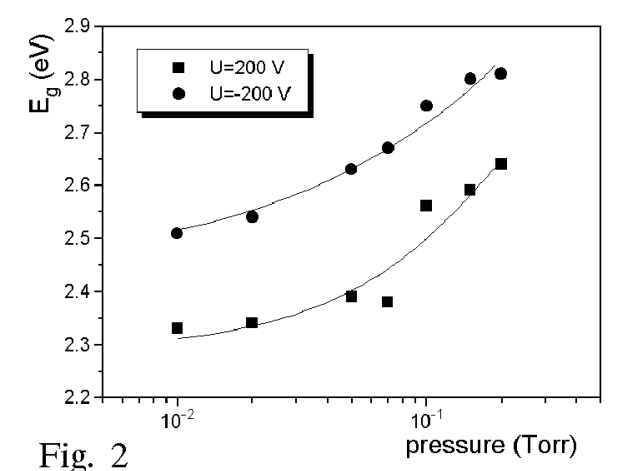

Fig. 2

Fig. 1. Transmission and reflection spectra for a sample with thickness of $1.81 \mu \mathrm{m}$, obtained in following conditions: working gas $\mathrm{Ar}: \mathrm{CH}_{4}=1: 1$, gas pressure $10^{-2}$ Torr and $-200 \mathrm{~V}$ bias voltage.

Fig. 2. The optical band gap of films as a function of the deposition pressure for two applied bias voltages.

band gap increases with increasing pressure. Since the band gap of graphite is zero and band gap of diamond is $5.5 \mathrm{eV}$, the increase in band gap with increasing pressure can be considered to be the result of transition from unsaturated graphitic $s p^{2}$ bonds to tetrahedral diamond-like $s p^{3}$ bonds in the film [21]. This can be clearly seen from the results in Fig. 3, which shows the band gap as a function of $s p^{3} / s p^{2}$ ratio. The increase in the band gap when the proportion of tetrahedral bondings increase is in agreement with published data [22]. The presence of $s p^{2}$ carbon is energetically correlated with a $\pi$ band inside the $\sigma$ band gap and leads to a decrease in the optical band gap when the graphitic part increases [23].

The slope of linear region on the Tauc plot (Eq. (1)), $G$, is a density-of-states coefficient (in $\mathrm{cm}^{-1 / 2} \mathrm{eV}^{-1 / 2}$ ) in direct proportion with the energy width of the localized states [24]. The values of $G$ commonly reported for amorphous carbon are in the range of $150-300 \mathrm{~cm}^{-1 / 2} \mathrm{eV}^{-1 / 2}$. For the films investigated here, the values

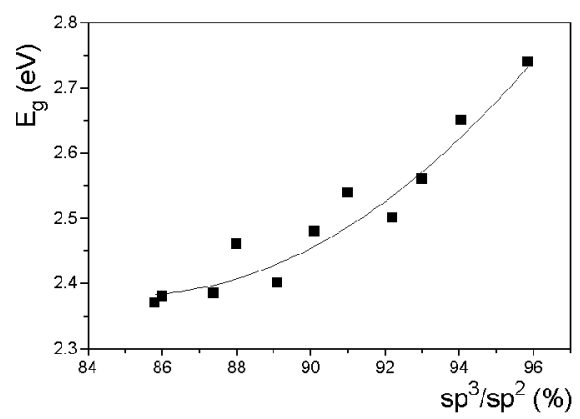

Fig. 3. Band gap as a function of $s p^{3} / s p^{2}$ ratio for films obtained under different gas pressures and different applied bias voltages. 


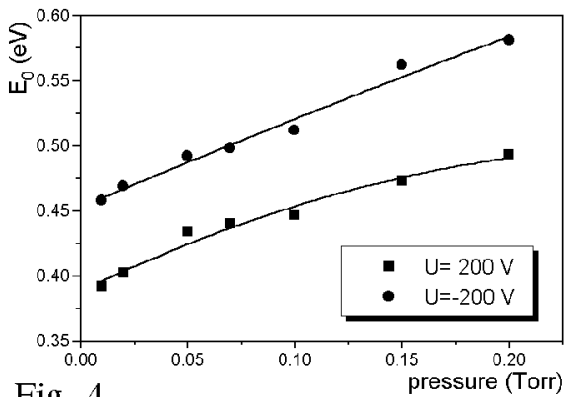

Fig. 4

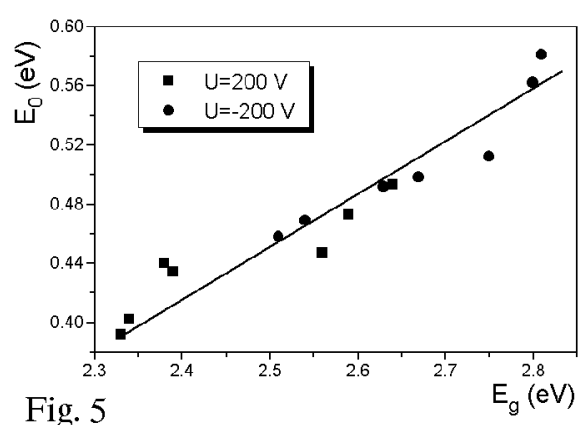

Fig. 5

Fig. 4. The Urbach energy as function of gas pressure, for two values of substrate applied bias voltage.

Fig. 5. The plot showing the relationship between the optical band gap $\left(E_{\mathrm{g}}\right)$ and the Urbach energy $\left(E_{0}\right)$ of the a-C:H films deposited at two applied bias voltages.

of $G$ were in the range of $150-200 \mathrm{~cm}^{-1 / 2} \mathrm{eV}^{-1 / 2}$, and that can be correlated with a low density of the localized states, in agreement with the relatively high values of the optical band gap.

It is known that the absorption edge (the Urbach region) in amorphous semiconductors is caused by the disorder in the film [25]. In the absorption constant range from $1 \mathrm{~cm}^{-1}$ (or less) to about $10^{4} \mathrm{~cm}^{-1}$, the $\alpha$ values can be fitted to

$$
\alpha=\alpha_{0} \exp \left(\frac{E}{E_{0}}\right)
$$

where $E_{0}$ is the Urbach energy. In Fig. 4 the Urbach energy as a function of gas pressure is shown, for two values of substrate applied bias voltage. The obtained values for the Urbach energy are in the inferior limit of the values reported for amorphous carbon, indicating a relative order in the layer. The Urbach energy increases with the deposition pressure, because of the decrease in the substrate incident species energy, favorizing the random linking of carbon atoms and increasing the disorder.

It is interesting to note that the plot of $E_{0}$ against $E_{\mathrm{g}}$ gives a linear relationship as shown in Fig. 5, similar with the results of Lee et al. [2]. Since $E_{0}$ depends on the disorder and $E_{\mathrm{g}}$ is correlated with the proportion of the graphite component, the decrease in the Urbach energy with the decrease in the optical band gap indicates that the trigonal links enhances the structural order in the films.

The main parameter of films which influences the refractive index is the density. Figure 6 and 7 shows the relationships between the refractive index and applied bias voltage (Fig. 6) for a deposition pressure $p=0.05$ Torr and between the refractive index and deposition pressure (Fig. 7), for two substrate applied bias voltages. The insets show the variation of the density against the applied bias voltage (for Fig. 6) and against deposition pressure (for Fig. 7). The refractive 


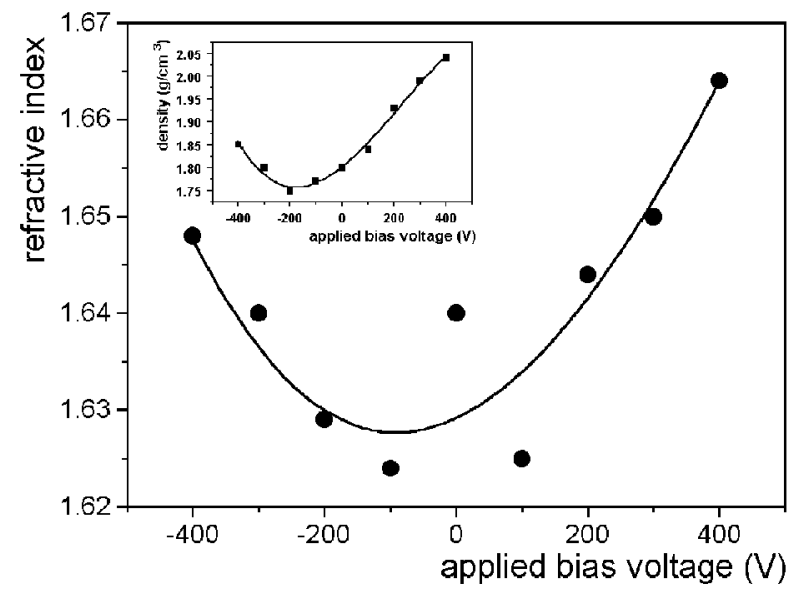

Fig. 6. Refractive index versus applied bias voltage for deposition gas pressure $p=0.05$ Torr. Inset of the figure shows the variation of the density with applied bias voltage.

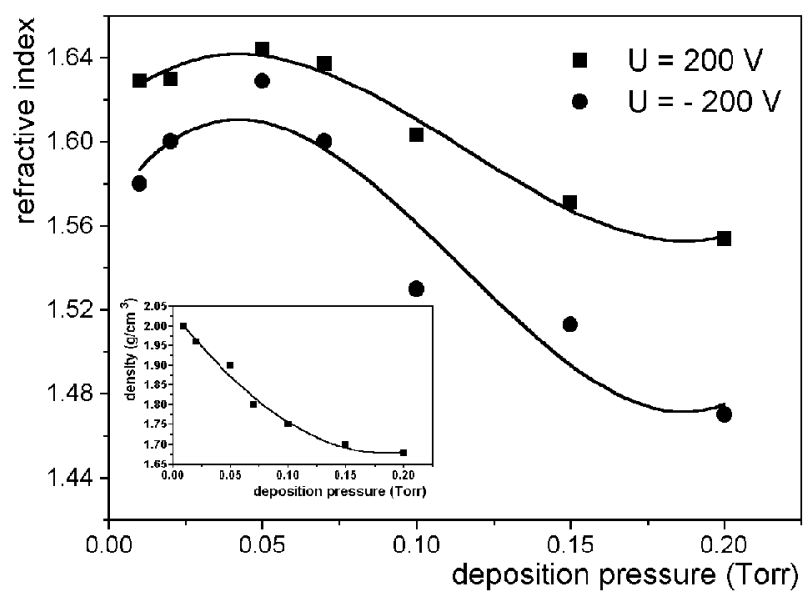

Fig. 7. Refractive index versus deposition gas pressure for two applied bias voltages. Inset of the figure shows the variation of the density with the deposition gas pressure.

index increases for both positive and negative biasing. When the gas pressure increases, the refractive index decreases. The inserts of both figures (6 and 7) reveal similar dependencies between density and the corresponding parameters. According to Lorenz-Lorentz equation relating to refractive index and density, an increase in the refractive index indicates an increase in the density of the film [26]. Figure 8 shows the relationship between the refractive index and film density and confirms the presumed behavior.

The refractive index is correlated also with the optical band gap. It is generally accepted that the refractive index of the amorphous carbon films decreases 


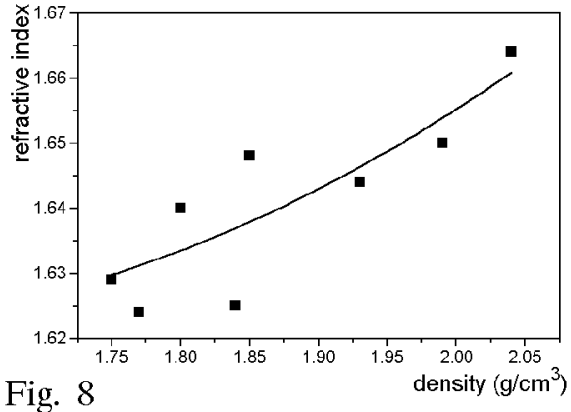

Fig. 8

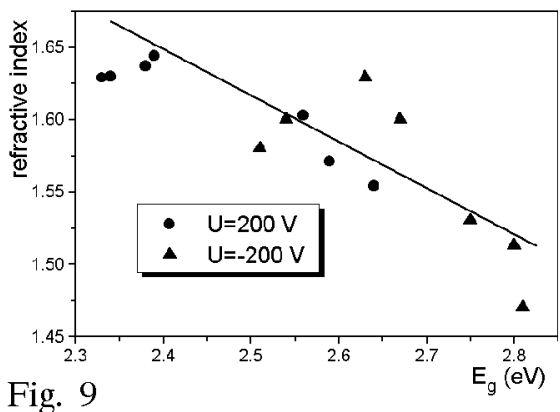

Fig. 9

Fig. 8. Refractive index as a function of density for films deposited at 0.05 Torr with different applied bias voltages.

Fig. 9. Refractive index as a function of the optical band gap.

when optical gap increases [27], in accordance with the founded relation between the refractive index and the optical band gap (Fig. 9).

\section{Conclusions}

In this paper the optical properties for rf magnetron sputtered a-C:H thin films are investigated for different applied bias voltages and gas pressures. The optical band gap of amorphous carbon increases with increasing pressure and that can be considered to be the result of transition from unsaturated graphitic $s p^{2}$ bonds to tetrahedral diamond-like $s p^{3}$ bonds in the film. The values of the slope of linear region on the Tauc plot $G$ can be correlated with a low density of the localized states, in agreement with the relatively high values of the optical band gap. The obtained values for the Urbach energy are in the inferior limit of the values reported for amorphous carbon, indicating a relative order in the layer. The decrease in the Urbach energy with the decrease in the optical band gap indicates that the trigonal links enhance the structural order in the films. The refractive index increases for both positive and negative biasing. When the gas pressure increases, the refractive index decreases. In both cases, the increase in the refractive index is correlated with the increase in films density.

\section{References}

[1] S.R.P. Silva, R.U.A. Khan, A.P. Burden, J.V. Anguita, J.M. Shannon, B.J. Sealy, A.J. Papworth, C.J. Kiely, G.A.J. Amaratunga, Thin Solid Films 332, 118 (1998).

[2] J.H. Lee, Y.H. Lee, B. Farouk, J. Appl. Phys. 79, 7676 (1996).

[3] R. Gago, O. Sanchez-Garrido, A. Climent-Font, J.M. Albella, E. Roman, J. Raisanen, E. Rauhala, Thin Solid Films 338, 88 (1999).

[4] Y. Inoue, T. Komoguchi, H. Nakata, O. Takai, Thin Solid Films 322, 41 (1998). 
[5] A. Bubenzer, B. Dischler, G. Brandt, P. Koidl, J. Appl. Phys. 54, 4590 (1983).

[6] F. Pawlak, Ch. Dufour, A. Laurent, E. Paumier, J. Perriere, J.P. Stoquert, M. Toulemonde, Nucl. Instrum. Methods Phys. Res. B 151, 140 (1999).

[7] J. Schwan, S. Ulrich, H. Roth, H. Ehrhardt, S.R.P. Silva, J. Robertson, R. Samlenski, R. Brenn, J. Appl. Phys. 79, 1416 (1996)

[8] Y. Taki, O. Takai, Thin Solid Films 316, 45 (1998).

[9] Shi Xu, L.K. Cheah, B.K. Tay, Thin Solid Films 312, 160 (1998).

[10] R.S. Brusa, A. Somoza, H. Huck, N. Tiengo, G.P. Karwasz, A. Zecca, M. Reinoso, E.B. Halac, Appl. Surf. Sci. 150, 202 (1999).

[11] D.L. Pappas, K.L. Saenger, J. Bruley, W. Krakow, J.J. Cuomo, Tieer Gu, R.W. Collins, J. Appl. Phys. 71, 5675 (1992).

[12] D.A. Anderson, Philos. Mag. 35, 17 (1977).

[13] E.H.A. Dekempeneer, R. Jacobs, J. Smeets, J. Meneve, L. Eersels, B. Blanpain, J. Roos, D.J. Oostra, Thin Solid Films 217, 56 (1992).

[14] L. Martinu, A. Raveh, A. Dominique, L. Bertrand, J.E. Klemberg-Sapieha, S.C. Gujrathi, M.R. Wertheimer, Thin Solid Films 208, 42 (1992).

[15] X. Jiang, J.W. Zou, C. Reichelt, P. Grunberg, J. Appl. Phys. 66, 4729 (1989).

[16] I. Vascan, I. Lazar, M. Stamate, G. Lazar, I. Rusu, SPIE Proceedings 2461, 611 (1995)

[17] I. Lazar, I. Vascan, Rom. J. Phys. 43, 557 (1998).

[18] M. Stamate, G.I. Rusu, E. Iordachescu, A. Stamate, I. Vascan, Analele Stiint. Univ. "Al.I. Cuza" Iasi (Ser. Noua) I.B. Fiz. XXXVIII, 548 (1993).

[19] R. Swanapoel, J. Phys. E 16, 1214 (1983).

[20] G. Lazar, I. Vascan, Rom. J. Phys. 43, 571 (1998).

[21] Z.F. Li, Z.Y. Yang, R.F. Xiao, J. Appl. Phys. 80, 5398 (1996).

[22] Shi Xu, L.K. Cheah, B.K. Tay, Thin Solid Films 312, 160 (1998).

[23] L.H. Chou, H.W. Wang, J. Appl. Phys. 74, 4673 (1993).

[24] F.M. El-Hossary, D.J. Fabian, A.P. Webb, Thin Solid Films 192, 201 (1990).

[25] J. Tauc, Amorphous and Liquid Semiconductors, Plenum, London 1974, p. 179.

[26] M. Nakayama, A. Tsuyoshi, M. Shibahara, K. Maruyama, K. Kamata, J. Vac. Sci. Technol. A 13, 195 (1995).

[27] P. Koidl, C. Wild, R. Chocher, R.E. Sah, Diamond and Diamond-like Films and Coatings, Vol. 266, Plenum, New York 1991, p. 243. 